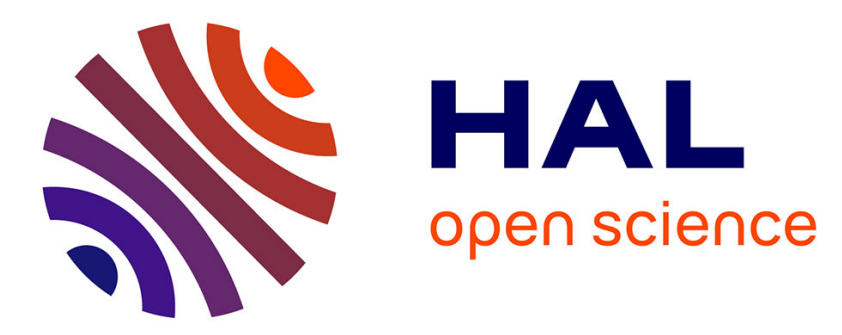

\title{
Etude comparative du claquage dans les transistors à effet de champ de puissance MESFET, TEGFET et MISFET
}

Jean-Claude de Jaeger, Farid Temcamani, M. . Lefebvre, R. Kozlowski, P. Fellon, J. Pribetich, Y. Crosnier

\section{To cite this version:}

Jean-Claude de Jaeger, Farid Temcamani, M. . Lefebvre, R. Kozlowski, P. Fellon, et al.. Etude comparative du claquage dans les transistors à effet de champ de puissance MESFET, TEGFET et MISFET. Revue de Physique Appliquée, 1988, 23 (7), pp.1205-1213. 10.1051/rphysap:019880023070120500 . jpa-00245932

\section{HAL Id: jpa-00245932 https://hal.science/jpa-00245932}

Submitted on 1 Jan 1988

HAL is a multi-disciplinary open access archive for the deposit and dissemination of scientific research documents, whether they are published or not. The documents may come from teaching and research institutions in France or abroad, or from public or private research centers.
L'archive ouverte pluridisciplinaire HAL, est destinée au dépôt et à la diffusion de documents scientifiques de niveau recherche, publiés ou non, émanant des établissements d'enseignement et de recherche français ou étrangers, des laboratoires publics ou privés. 


\title{
Etude comparative du claquage dans les transistors à effet de champ de puissance MESFET, TEGFET et MISFET
}

\author{
J. C. de Jaeger, F. Temcamani, M. Lefebvre, R. Kozlowski, P. Fellon, J. Pribetich et Y. Crosnier \\ Centre Hyperfréquences et Semiconducteurs, U.A. 287 C.N.R.S., Bât. P4, Université des Sciences et \\ Techniques de Lille Flandres Artois, 59655 Villeneuve d'Ascq Cedex, France
}

(Reçu le 16 octobre 1987, révisé le 24 novembre 1987, accepté le 8 décembre 1987)

\begin{abstract}
Résumé. - Une étude comparative concernant la tension de claquage au pincement dans les transistors à effet de champ MESFET GaAs, TEGFET AlGaAs/GaAs et MISFET InP est présentée. Elle comporte une analyse théorique réalisée à partir de deux modélisations bidimensionnelles reposant sur une résolution rigoureuse des équations physiques des semiconducteurs. La première, relativement simple, traite l'apparition du claquage en absence de courant alors que la seconde très complète tient compte des courants d'électrons et de trous. Cette étude est confortée par de nombreux résultats expérimentaux et permet de faire le point sur l'influence des principaux paramètres des différentes structures. Elle apporte également des éléments de compréhension concernant les effets liés au recess de grille, à la distance et à la configuration de l'espace grille-drain et enfin aux profils de dopage particuliers sous la grille.

Abstract. - A study about breakdown voltage near pinchoff in GaAs MESFET's, AlGaAs/GaAs TEGFET's and InP MISFET's is described. The theoretical analysis is made using two dimensional models based on a complete calculation of semiconductor physical equations. The first model, the easier, determine the breakdown voltage without current. On the other hand, the second model is more accurate and takes into account the electron and hole currents. This study is in good agreement with many experimental results and makes it possible to report the effect of the main parameters of the different structures. The problems due to a gate recess, the length and shape of gate-drain zone and, at last, particular doping concentration layers underneath the gate are also investigated.
\end{abstract}

\section{Introduction.}

La tension de claquage des transistors à effet de champ constitue une limitation fondamentale à la production de puissance par ces composants. Le problème constitue une préoccupation permanente dans les travaux que poursuit depuis plusieurs années notre laboratoire sur les transistors à effet de champ de puissance. Une première étude, portant sur le MESFET GaAs nous a conduit, il y a déjà quelques années, à une première description [1] des mécanismes de claquage basée sur une modélisation incluant de nombreux phénomènes physiques tels que la dynamique électronique non stationnaire, l'ionisation des porteurs et les effets bidimensionnels liés aux bords de grille. Ce premier modèle nous avait permis d'apporter une approche vraiment physique des différents aspects du claquage dans les MESFETs
GaAs dont les conclusions essentielles se sont vues corroborées par les travaux de Frensley [2], de Wemple [3] et, très récemment, par ceux de Zaitlin [4]. Cette première étude ayant mis en évidence l'extrême importance des effets liés à la nature bidimensionnelle des structures, il nous a paru indispensable de développer d'autres modèles tenant plus rigoureusement compte de cet impératif et suffisamment généraux pour être adaptables facilement à des structures très variées, en particulier celles du TEGFET AlGaAs/GaAs et du MISFET InP qui font, actuellement, l'objet de nombreuses études pour leurs potentialités en tant que composants microondes de puissance. Nous avons ainsi mis sur pieds deux modélisations rigoureusement bidimensionnelles, l'une, relativement légère, traitant seulement le seuil de tension d'apparition du claquage et l'autre très complète et nécessairement plus 
lourde, décrivant entièrement le mécanisme du claquage dans toute son étendue, en présence de courant.

\section{Modélisation bidimensionnelle du claquage en configuration diode.}

Cette modélisation a deux hypothèses simplificatrices : l'absence totale de courant et des potentiels égaux sur source et drain. Dans la pratique, le claquage ainsi décrit correspond à la configuration diode entre grille, d'une part, et source et drain court-circuités, d'autre part. Cette configuration n'est pas exactement celle que l'on a en véritable polarisation transistor où source et drain sont à des potentiels différents. Néanmoins, l'expérience pratiquée sur de nombreux composants nous a montré que la tension de claquage obtenue en configuration diode est généralement assez voisine de la tension grille-drain de claquage en configuration transistor, au pincement. Cette modélisation nous est donc apparue pouvant donner avec une bonne approximation le seuil de l'apparition du claquage au pincement en polarisation normale transistor.

Quelle que soit la structure, elle est représentée sous la forme du modèle de la figure 1 où la symétrie par rapport à l'axe vertical $O y$ traduit l'hypothèse des potentiels égaux sur la source et le drain.

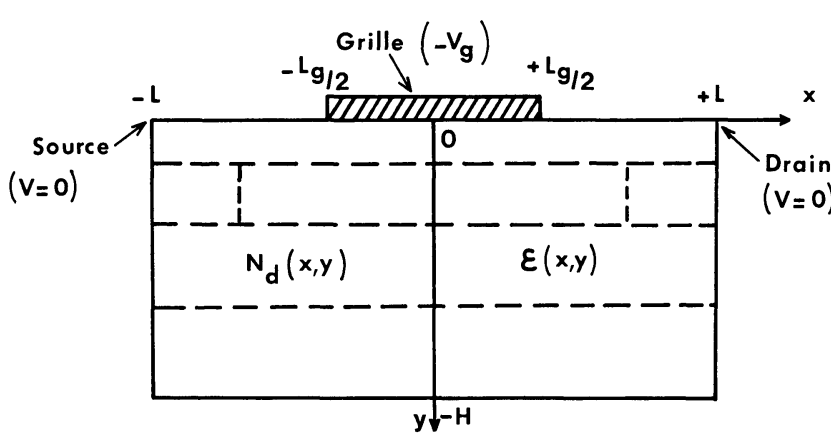

Fig. 1. - Modélisation bidimensionnelle du transistor à effet de champ, en absence de courant, utilisée pour le calcul de la tension de claquage $\left(V_{\mathrm{Ds}}=0\right)$.

[Two dimensional model description of the field effect transistor, without current, used for computing breakdown voltage $\left(V_{\mathrm{DS}}=0\right)$.]

Les différentes couches et régions sont délimitées horizontalement et verticalement en zones ayant leur propre dopage $N_{\mathrm{D}}(x, y)$ et leur propre constante diélectrique $\varepsilon(x, y)$. Le calcul du potentiel $V(x, y)$ en tout point de la structure s'effectue en résolvant simultanément :

* l'équation de Poisson

$$
\begin{aligned}
\frac{\delta^{2} V}{\delta x^{2}}+\frac{\delta^{2} V}{\delta y^{2}}= & -q \frac{\left(N_{\mathrm{D}}-n\right)}{\varepsilon}-\frac{1}{\varepsilon} \frac{\delta \varepsilon}{\delta x} \frac{\delta V}{\delta x}- \\
& -\frac{1}{\varepsilon} \frac{\delta \varepsilon}{\delta y} \frac{\delta V}{\delta y}
\end{aligned}
$$

* l'équation du courant

$$
\mathrm{J}=-q n \mu \operatorname{grad} V+q D_{\mathrm{n}} \operatorname{grad} n=0
$$

où $V, N_{\mathrm{D}}, n, \varepsilon$ sont respectivement le potentiel, le dopage, la concentration d'électrons libres et la permittivité diélectrique en un point du maillage de coordonnées $x, y$. La combinaison de ces deux équations aboutit à une équation non linéaire qui est résolue par la méthode de Newton-Raphson à deux dimensions en prenant pour conditions aux limites (Fig. 1) :

$$
\begin{array}{lll}
V=V_{\mathrm{G}} & \text { pour } & y=0 \text { et } 0<|x|<L_{\mathrm{G}} / 2 \\
V=0 & \text { pour } x= \pm L \text { et } y=0
\end{array}
$$

$\delta V / \delta x=0$ immédiatement sous la grille pour tenir compte de sa nature conductrice.

$\delta V / \delta x=0$ sur l'axe $O y$, par symétrie du modèle. $\delta V / \delta y=0$ pour $y=0$ et $L_{\mathrm{G}} / 2<|x|<L$ pour traduire le contraste élevé de permittivité diélectrique entre le semiconducteur et le milieu extérieur.

On obtient ainsi la configuration des équipotentielles. Un exemple typique en est donné sur la figure 2 dans le cas d'un MISFET InP où la moitié seulement de la structure a été représentée. La zone de dopage $N_{\text {D1 }}$ représente la couche active, celle de dopage $N_{\mathrm{D} 3}$ la zone $\mathrm{N}^{+}$de contact ohmique de source ou de

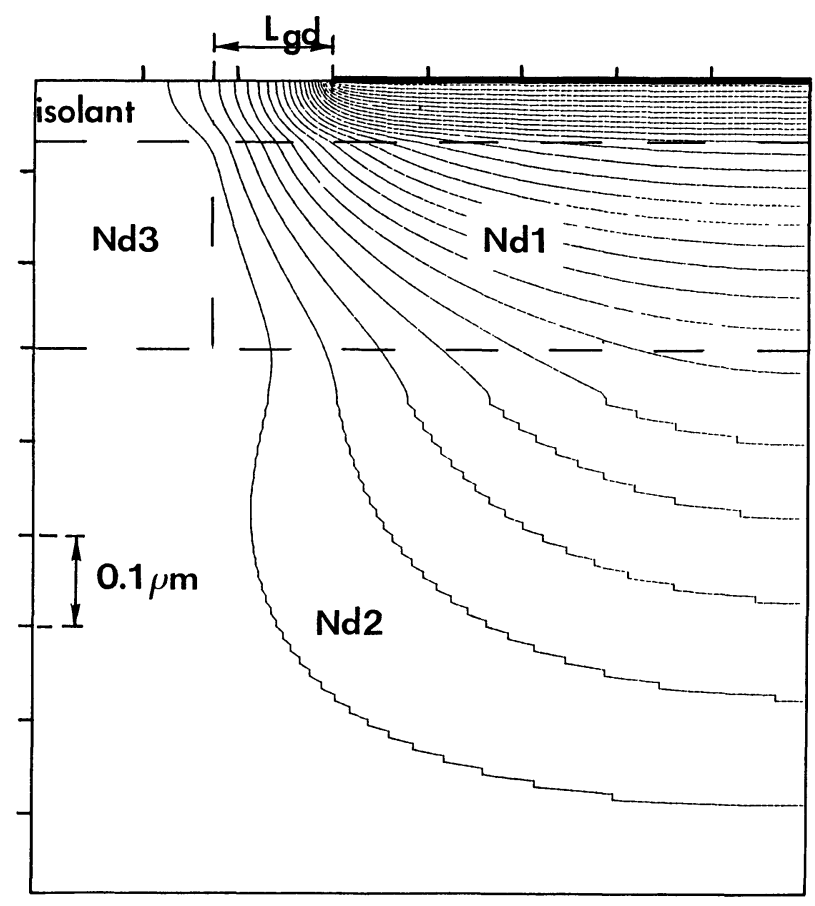

Fig. 2. - Exemple d'équipotentielles obtenues avec le modèle bidimensionnel, en absence de courant, dans le cas du MISFET InP.

[Example of equipotential shape calculated with the two dimensional model, without current, for the InP MISFET's.] 
drain et enfin la zone de dopage $N_{\mathrm{D} 2}$ le substrat. Les équipotentielles sont ici représentées pour une tension proche du claquage avec un pas de $1 \mathrm{~V}$. On peut notamment remarquer que celles-ci se resserrent dans l'isolant ce qui implique une augmentation du champ électrique dans cette zone. La raison physique de cet effet est la faiblesse de permittivité diélectrique de l'isolant devant celle du semiconducteur.

A partir du potentiel en chaque point on détermine les lignes de champ électrique. Le calcul de la condition d'avalanche a été traité en prenant pour hypothèse simplificatrice que les coefficients d'ionisation des électrons et des trous sont sensiblement égaux. Le coefficient d'ionisation $\alpha$ est pris de la forme :

$$
\alpha=A \exp \left[-(b / E)^{m}\right]
$$

où $A, b$ et $m$ dépendent du milieu considéré.

La dépendance de $\alpha$ par rapport au champ électrique $E$ a été prise conformément aux résultats usuels donnés dans la littérature pour les différents semiconducteurs envisagés : GaAs [5], AlGaAs [6] et InP [7]. La figure 3 montre les lois $\alpha(E)$ utilisées pour ces trois matériaux. On peut y constater que, sauf pour les champs électriques élevés, l'AlGaAs est le matériau le moins ionisant et le GaAs le plus

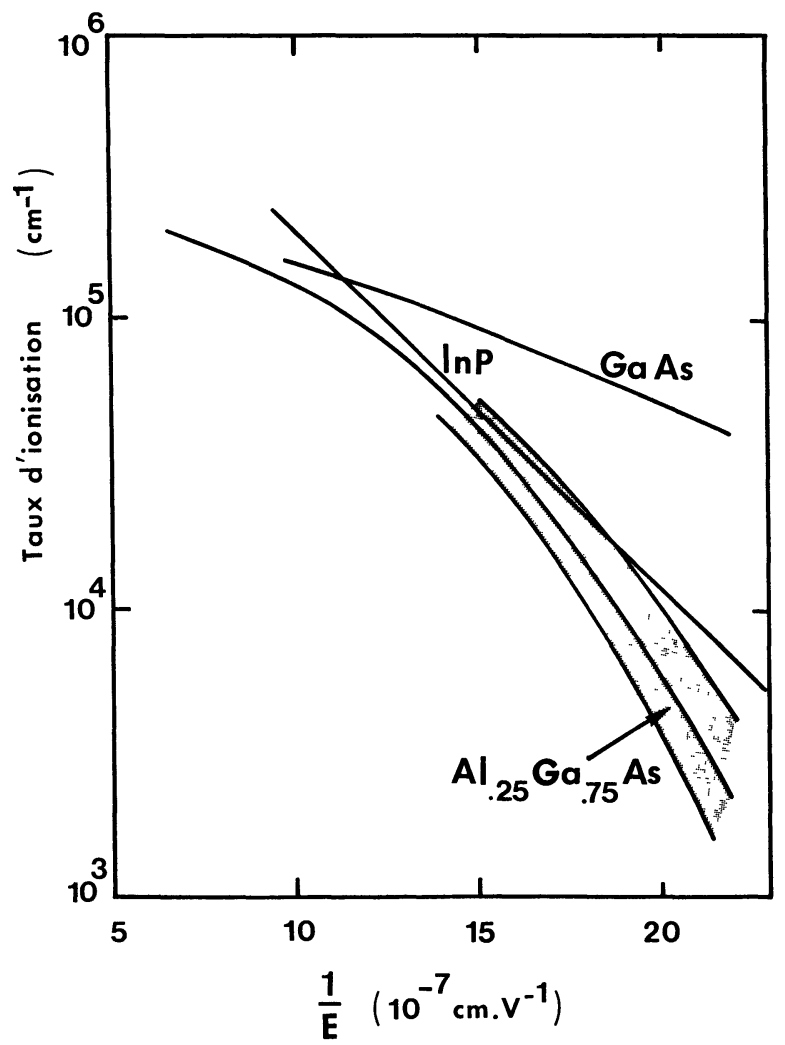

Fig. 3. - Evolution des coefficients d'ionisation en fonction du champ électrique pour le GaAs, l'InP et le AlGaAs.

[Electric field dependence of ionization coefficients for GaAs, InP and AlGaAs.] ionisant. En ce qui concerne l'isolant de la structure MISFET InP, nous avons utilisé une formulation analogue (Eq. (3)) avec les paramètres relatifs au $\mathrm{SiO}_{2}[8]$ :

$$
\begin{aligned}
A & =3,3 \times 10^{6}\left(\mathrm{~cm}^{-1}\right) \\
b & =78 \times 10^{6}(\mathrm{~V} / \mathrm{cm}) \\
m & =1 .
\end{aligned}
$$

\section{Modélisation bidimensionnelle du claquage en configuration transistor.}

Ce modèle est constitué comme dans le cas du $1^{\text {er }}$ modèle à partir d'un contour (Fig. 1) dont l'intérieur est repéré par un maillage. Il découle directement de celui mis au point au laboratoire pour'les MESFETs à grille submicronique [9]. Il s'agit d'un traitement physique très rigoureux basé sur la résolution de l'équation de Boltzmann. Les équations utilisées sont :

* L'équation de Poisson :

$$
\Delta V=\frac{q}{\varepsilon_{0} \varepsilon_{\mathrm{r}}}\left(n-p-N_{\mathrm{D}}\right)
$$

* Les équations de continuité pour les électrons et les trous :

$$
\begin{aligned}
& \frac{\delta n}{\delta t}=\frac{1}{q} \operatorname{div} \mathbf{J}_{\mathrm{n}}+g \\
& \frac{\delta p}{\delta t}=-\frac{1}{q} \operatorname{div} \mathbf{J}_{\mathrm{p}}+g
\end{aligned}
$$$$
\text { avec } \quad g=\alpha\left(\left|v_{\mathrm{n}}\right| n+\left|v_{\mathrm{p}}\right| p\right)
$$

* L'équation de relaxation de l'énergie :

$$
\frac{\delta n w}{\delta t}=\mathbf{J}_{\mathrm{n}} \mathbf{E}+\operatorname{div} \mathbf{J}_{\mathrm{n}} w-\frac{n w}{\tau_{w}} .
$$

* Les équations des courants d'électrons et de trous :

$$
\begin{aligned}
& \mathbf{J}_{\mathrm{n}}=q \mu_{\mathrm{n}}\left(n \mathbf{E}+\operatorname{grad} \frac{n k T_{\mathrm{n}}}{q}\right) \\
& \mathbf{J}_{\mathrm{p}}=q \mu_{\mathrm{p}}\left(p \mathbf{E}-\operatorname{grad} \frac{p k T_{\mathrm{p}}}{q}\right)
\end{aligned}
$$

où $n, p, N_{\mathrm{D}}$ représentent les concentrations d'électrons, de trous et le dopage

$g$ est un terme de génération par ionisation.

$w$ est l'énergie des électrons et $\tau_{w}$ le temps de relaxation de celle-ci.

$T_{\mathrm{n}}$ et $T_{\mathrm{p}}$ sont les températures des électrons et des trous.

$v_{\mathrm{n}}$ et $v_{\mathrm{p}}$ sont les vitesses des électrons et des trous.

L'avalanche est introduite dans ces équations par la présence du terme $g$ de génération par ionisation et par l'existence d'un courant grille particulaire. En ce qui concerne les conditions aux limites, on utilise sur 
les surfaces limites du semiconducteur les conditions de Neumann :

$$
\frac{\delta V}{\delta \eta}=0 \quad \frac{\delta n}{\delta \eta}=0 \quad \frac{\delta w}{\delta \eta}=0
$$

où $\eta$ est la normale à la surface.

Sur les électrodes on utilise les conditions de Dirichlet :

$V=$ Cste

$w=w_{0}$ (énergie moyenne correspondant à la température $T_{0}$ )

$n=N_{\mathrm{D}}$ (sur les contacts de source et de drain)

et on impose sur la grille la nullité du courant d'électrons mais l'existence du courant de trous. Malgré son temps calcul relativement modeste ( 23 min par point de polarisation sur IBM 3090), cette modélisation est d'une mise en œuvre assez lourde. Aussi, nous l'utilisons essentiellement comme moyen de validation de la modélisation la plus simple et surtout comme outil d'analyse permettant de comprendre finement les mécanismes de la mise en avalanche en présence de courant et le rôle joué par celui-ci.

Les figures 4 et 5 donnent des exemples typiques de résultats obtenus avec cette modélisation dans le cas d'un MESFET GaAs. La figure 4 montre pour

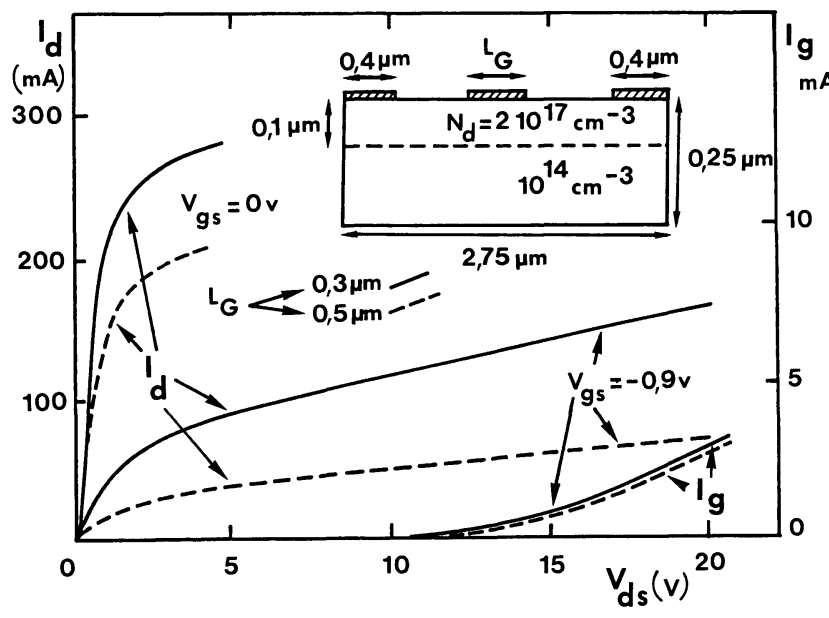

Fig. 4. - Evolutions théoriques des courants drain $I_{\mathrm{D}}$ et grille $I_{\mathrm{G}}$ en fonction de la tension $V_{\mathrm{DS}}$, obtenues pour un MESFET avec la modélisation bidimensionnelle complète (pour deux longueurs de grille).

[Theoretical drain current $I_{\mathrm{D}}$ and gate current $I_{\mathrm{G}}$ against $V_{\text {Ds }}$ voltage for a MESFET, computed with the complete two dimensional model (for two gate lengths).]

deux longueurs de grille différentes, 0,3 et $0,5 \mu \mathrm{m}$, les caractéristiques statiques obtenues $I_{\mathrm{D}}=$ $f\left(V_{\mathrm{DS}}, V_{\mathrm{GS}}\right)$ et les évolutions correspondantes du courant grille $I_{\mathrm{G}}$. La figure 5 donne pour la longueur de grille $0,3 \mu \mathrm{m}, V_{\mathrm{GS}}=-0,9 \mathrm{~V}$ et $V_{\mathrm{DS}}=20 \mathrm{~V}$, comment sont répartis dans la structure, le potentiel,
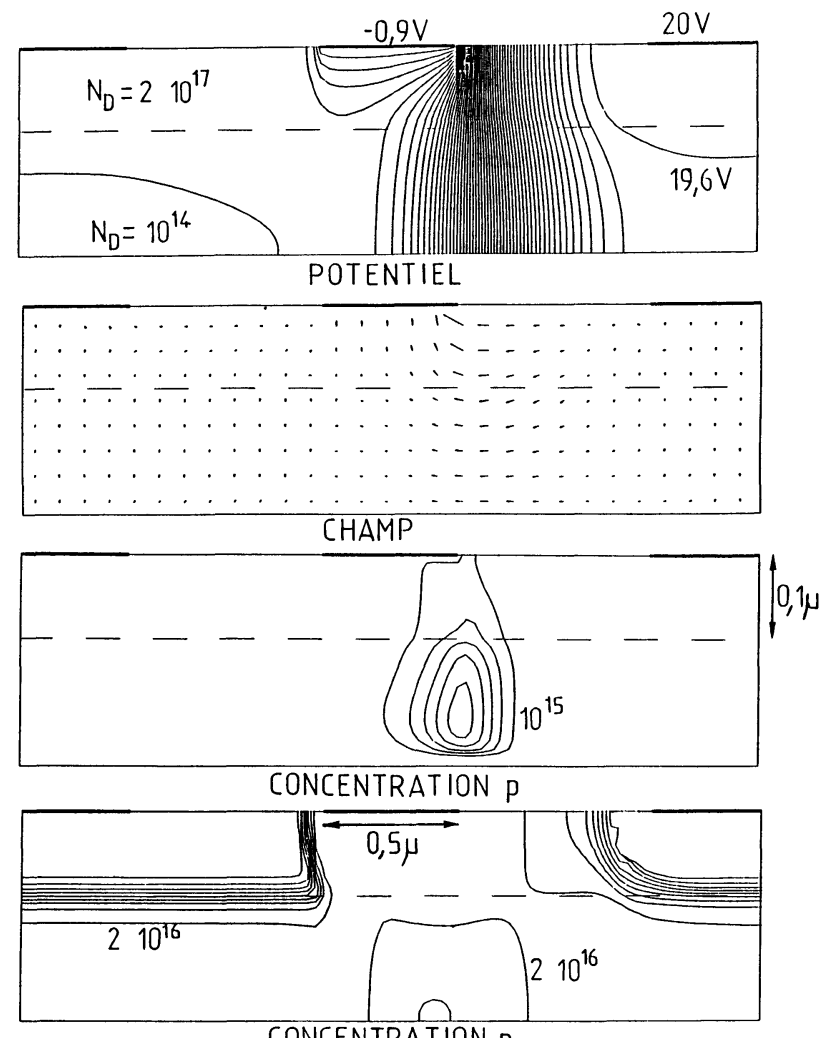

CONCENTRATION

Fig. 5. - Configuration des équipotentielles, du champ électrique et des concentrations de trous et d'électrons, au pincement, pour un MESFET.

A [Two dimensional dependence of equipotential lines, electric field and hole and electron concentrations, at pinchoff, for a MESFET.]

le champ électrique, les concentrations de trous et d'électrons. On peut y remarquer que la génération de trous s'opère effectivement dans la zone où règne un champ électrique élevé, c'est-à-dire dans l'espace grille drain, mais il est intéressant de noter que la concentration de trous générés par ionisation n'est pas maximum au voisinage immédiat du bord de grille, là où le champ électrique est le plus élevé, mais en profondeur. Cette situation qui pourrait $a$ priori surprendre a une explication physique liée au mécanisme même de génération : le terme $g$ intervenant dans les équations précédentes est, en effet, proportionnel au coefficient d'ionisation $\alpha$ mais aussi à la concentration locale de porteurs libres ; or, dans le cas présent de polarisation, la zone déplétée de grille s'étend suffisamment en profondeur pour repousser vers le substrat le passage du courant et par conséquent localiser plutôt en profondeur qu'en surface, la génération par ionisation.

\section{Aspects principaux du claquage dans les MES- FETs, TEGFETs et MISFETs.}

Quel que soit le type de transistor considéré, on observe un comportement du claquage qui évolue de 
façon similaire en fonction des principaux paramètres. Les figures $6 a, 6 b$ et $6 c$ donnent une comparaison des évolutions des tensions de claquage théoriques et expérimentales en fonction du dopage $N_{\text {D }}$ et de l'épaisseur de la couche qui pour chacun de ces composants est essentielle puisqu'elle constitue un réservoir de porteurs pour réaliser le canal source-drain. Pour le MESFET, c'est la couche de GaAs dite active, qui surmonte le substrat semiisolant. Pour le TEGFET, c'est la couche de AlGaAs surmontant la couche de GaAs non intentionnellement dopée qui, elle-même, surmonte le semi-isolant. Pour le MISFET, c'est la couche d'InP placée sous l'isolant de grille et surmontant le substrat semiisolant.

Sur le plan théorique, les évolutions générales sont similaires qualitativement pour les trois structures : la tension de claquage diminue en fonction du

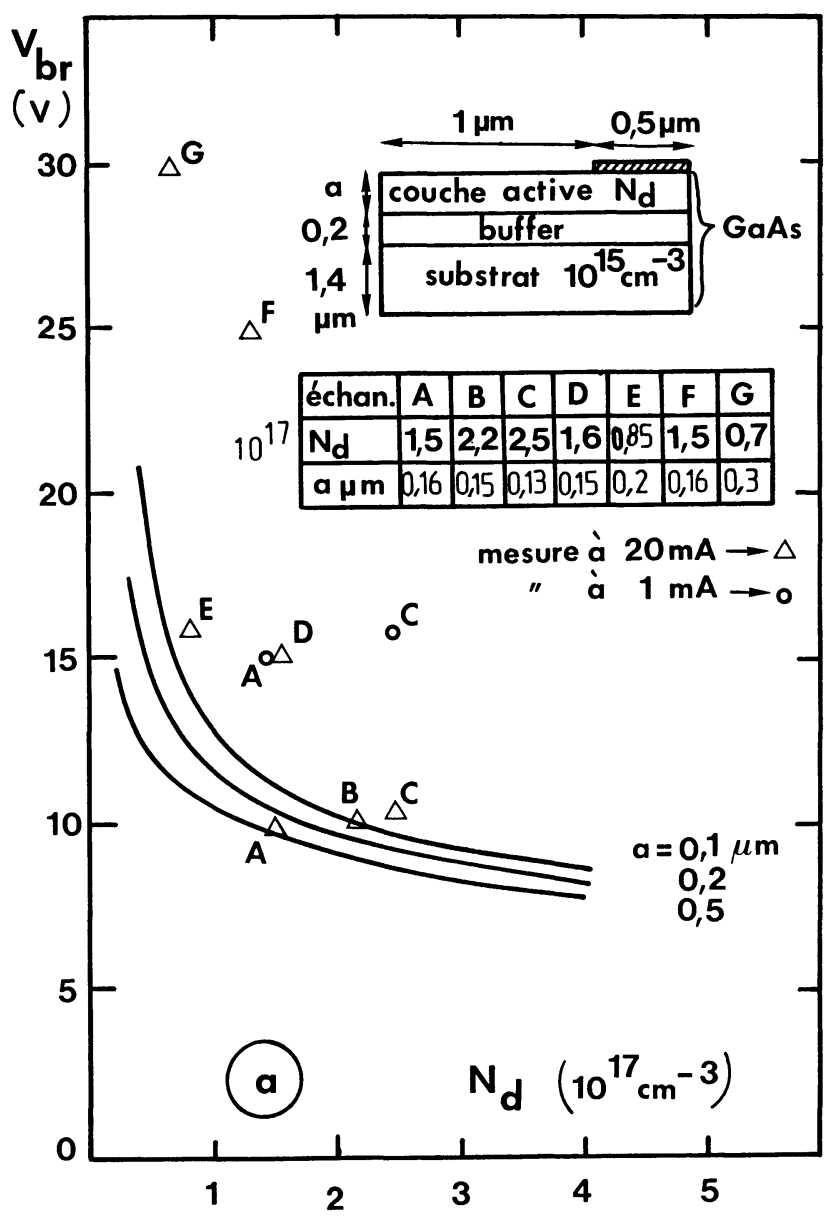

Fig. 6. - Résultats théoriques et expérimentaux de la tension de claquage en fonction du dopage et de l'épaisseur de couche pour le MESFET (a), le TEGFET (b) et le MISFET (c).

[Theoretical and experimental dependence of breakdown voltage versus the doping concentration and the depth of the layer for MESFET's (a), TEGFET's (b) and MISFET's (c).]

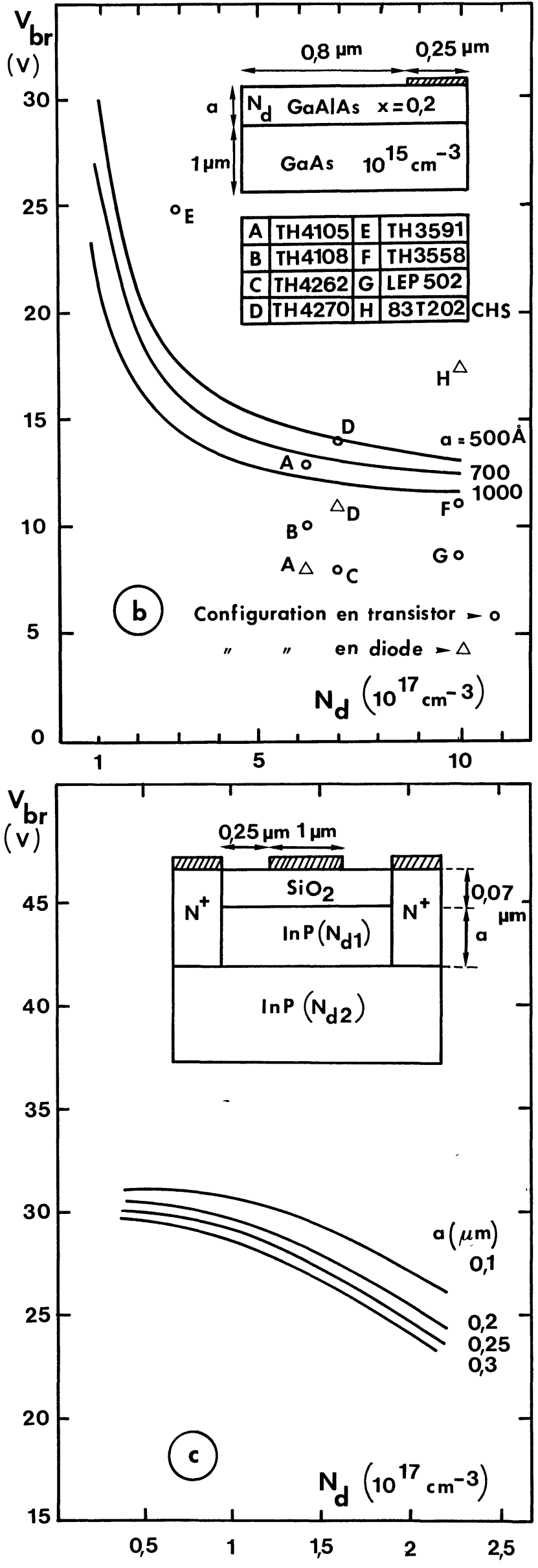


dopage $N_{\mathrm{D}}$ et de l'épaisseur $a$. L'observation de la configuration des lignes de champ électrique montre que, dans les trois cas, le claquage apparaît essentiellement au voisinage immédiat du bord de la grille. C'est en cet endroit que le champ électrique est toujours maximum et atteint des valeurs très élevées correspondant à la quasi-saturation du coefficient d'ionisation. A titre d'exemple, la figure 7 montre comment se présente typiquement cette situation dans le cas d'une structure MISFET InP. La figure 7a représente la configuration des lignes de champ pour $N_{\mathrm{D}}=5 \times 10^{16} \mathrm{~cm}^{-3}$ et $a=0,09 \mu \mathrm{m}$; la ligne en trait continu étant celle sur laquelle la condition d'avalanche est réalisée en premier. La figure $7 \mathrm{~b}$ permet de voir ce qu'il advient de la localisation de cette ligne de champ pour une épaisseur $a$ de $0,23 \mu \mathrm{m}$ et un dopage $N_{\mathrm{D}}$ de $10^{17} \mathrm{~cm}^{-3}$. Pour en revenir à la comparaison des trois structures, c'est pour le MESFET que les valeurs de tensions de
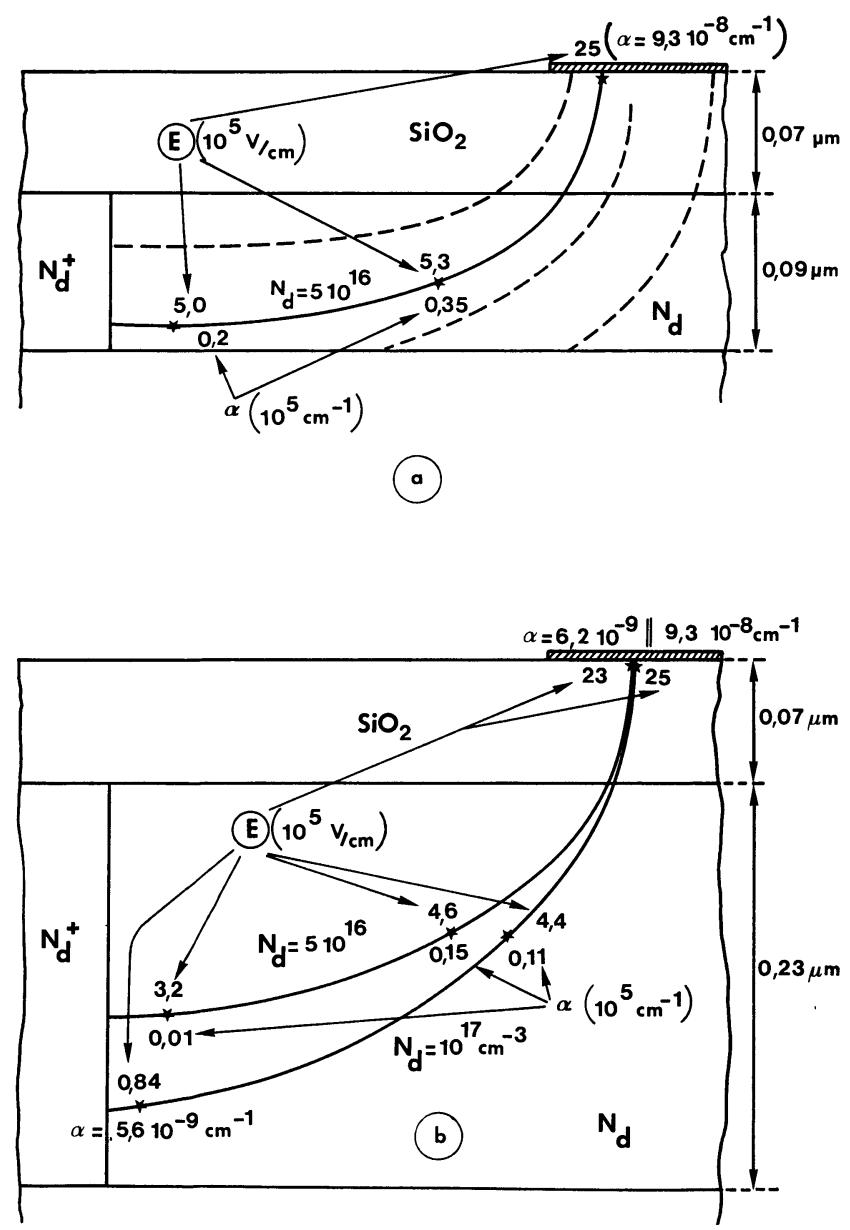

Fig. 7. - Lignes de champ électrique correspondant à la condition d'avalanche dans le cas du MISFET InP pour deux dopages et deux épaisseurs de la couche active.

[Electric field lines corresponding to avalanche breakdown for InP MISFET's for two doping concentrations and two channel thickness.] claquage sont les plus faibles. Elles sont globalement plus élevées de quelques volts pour le TEGFET. Ce résultat peut sembler normal étant donné que le coefficient d'ionisation de l'AlGaAs, est à champ électrique identique, pratiquement toujours inférieur à celui du GaAs seul (Fig. 3). L'utilisation dans le TEGFET de dopage nettement plus grand (presque une décade), en augmentant fortement le gradient du champ, aurait pu contrecarrer cet avantage. Ce n'est pas le cas.

En ce qui concerne le MISFET, les tensions de claquage sont encore plus élevées. Deux avantages essentiels sont à l'origine de cette supériorité : l'isolant supporte une partie importante de la tension et joue un rôle de tampon n'existant pas dans les structures MESFET et TEGFET. D'autre part, compte-tenu des coefficients d'ionisation utilisés dans cette étude, l'ionisation se produit dans l'InP, matériau moins ionisant.

Sur le plan expérimental, seuls les MESFETs et TEFGETs ont été étudiés, à partir de nombreux échantillons provenant de divers constructeurs. $\mathrm{Si}$, comme le montrent les figures $6 \mathrm{a}$ et $6 \mathrm{~b}$, l'accord avec les valeurs théoriques est globalement satisfaisant, on note cependant une certaine dispersion des tensions de claquage expérimentales. Celle-ci doit, sans doute, être attribuée au caractère idéal de la structure planaire utilisée pour la modélisation. Les structures réelles des composants testés sont en effet souvent plus complexes, en particulier à cause des recess de grille de géométrie très variable.

D'autre part, en ce qui concerne le MESFET GaAs, on peut noter que la plupart des points expérimentaux donnent une tension de claquage plus favorable que celle déterminée théoriquement. Ceci peut s'expliquer par le fait que nous n'avons pas pris en compte l'existence d'un potentiel de surface entre grille et drain dont l'influence n'est pas négligeable dans le cas du GaAs. Celle-ci provoque la création d'une zone désertée superficielle qui peut modifier notablement la répartition globale du potentiel entraînant une meilleure évolution du champ électrique dans l'espace grille-drain [10].

\section{Autres aspects du claquage dans les MESFETs, les TEGFETs et les MISFETs.}

5.1 PRoblème DU RECESS DE GRILlE. - De nombreuses mesures effectuées sur des MESFETs et TEGFETs à formes variables de recess de grille nous ont montré que le recess encaissé est de manière générale défavorable pour la tension de claquage et qu'il y a intérêt à avoir un recess relativement large voire graduel. Cet effet s'explique aisément à partir des configurations d'équipotentielles données par la modélisation avec courant. A titre d'exemple, la figure 8, concernant une structure de MESFET, montre clairement que la tension grille-drain se 
$0.3 \mu \mathrm{m}$

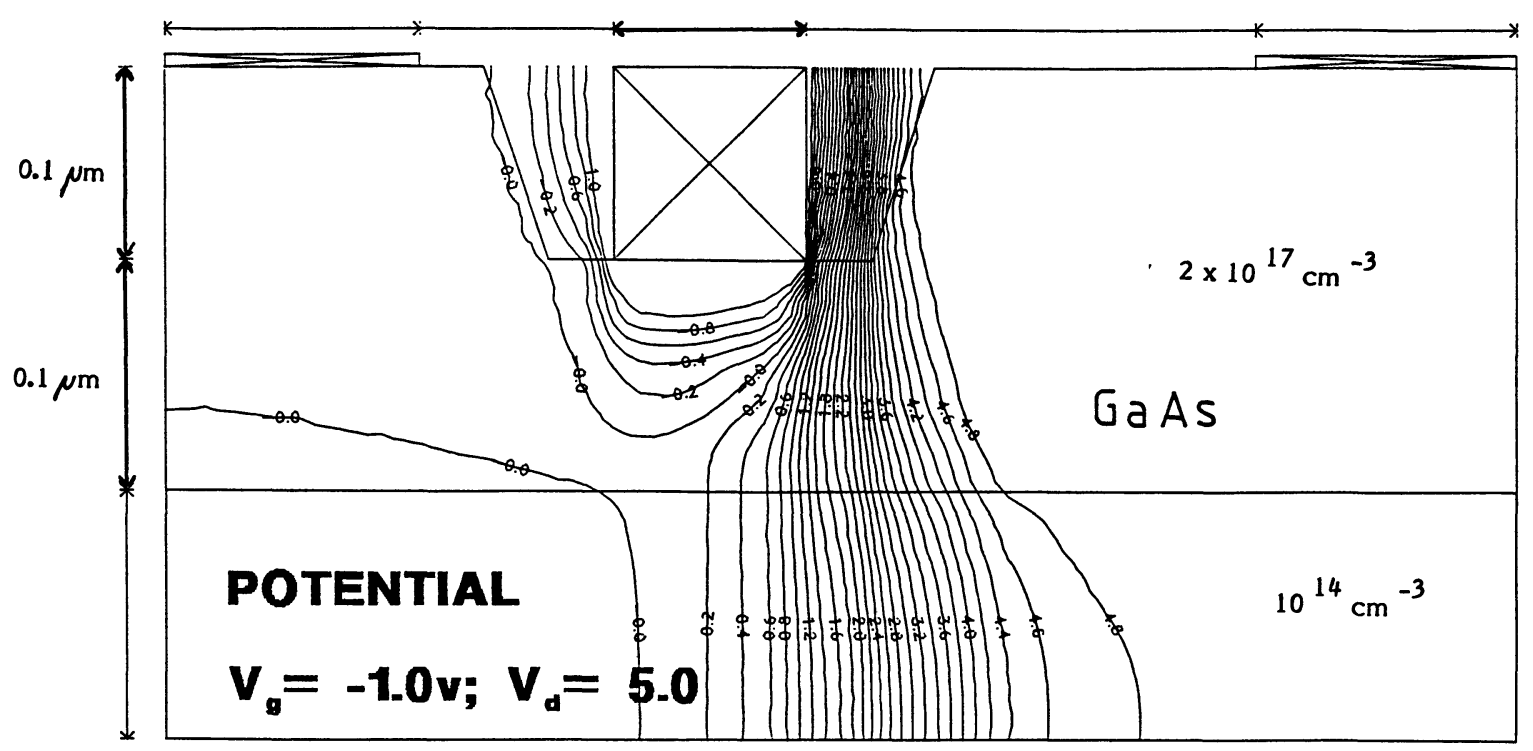

Fig. 8. - Configuration d'équipotentielles obtenues, au pincement, pour un MESFET présentant un recess de grille.

[Equipotential configuration at pinchoff, for a MESFET with a gate recess.]

trouve en effet entièrement concentrée dans le faible espace séparant la métallisation de grille du flanc du recess. On comprend aisément dès lors l'extrême importance de la géométrie de l'intervalle entre métallisation de grille et semiconducteur. L'étude quantitative de la tension de claquage en fonction de cette géométrie de recess est en cours avec cette modélisation.

\subsection{PROBlème DE LA DISTANCE GRILLE-DRAIN} $I_{\mathrm{gd}}$ - - Pour le MESFET et le TEGFET l'influence de cette distance est secondaire devant celle du recess lorsqu'une couche superficielle $\mathrm{N}^{+}$met pratiquement le contact de drain au voisinage immédiat de la grille. Des essais systématiques pratiqués sur des MESFETs de longueur de grille $l_{\mathrm{g}} \# 0,8 \mu \mathrm{m}$ avec $l_{\mathrm{gd}}$ variable $(6 \mu \mathrm{m}, 3 \mu \mathrm{m}$ et $1,5 \mu \mathrm{m})$ nous ont montré que cette distance n'influe pas sur la tension de claquage lorsqu'elle est supérieure à environ $1 \mu \mathrm{m}$, la tension de claquage relevée entre grille et drain source en l'air $\left(I_{\mathrm{D}}=1 \mathrm{~mA} / \mathrm{mm}\right)$ sur ces différents échantillons variant entre 17 et $19 \mathrm{~V}$.

Lorsque ce surdopage n'est pas superficiel mais seulement localisé sous le contact de drain, la configuration de l'espace grille-drain peut prendre de l'importance. C'est typiquement le cas du MESFET bigrille pour lequel l'existence d'une zone déplétée due à la seconde grille permet d'obtenir une tension de claquage plus élevée grâce à une meilleure répartition de la tension entre la première grille et le drain. Nous avons pu vérifier expérimentalement cette propriété en comparant des MES-
FETs monogrilles et des MESFETs bigrilles de topologies similaires élaborés à partir de la même structure de couches. Cette comparaison illustrée par les figures 9a et 9b montre clairement le comportement plus favorable du MESFET bigrille.

Les structures MISFET présentent également une certaine sensibilité du claquage en fonction de la distance grille drain $l_{\mathrm{gd}}$. Une illustration en est donnée sur la figure 10 . On peut y remarquer que lorsque $l_{\mathrm{gd}}$ devient supérieure à $0,5 \mu \mathrm{m}$ sa valeur n'a pratiquement plus d'influence sur la tension de claquage qui atteint alors sa valeur maximum.

\subsection{PROFIL DE DOPAGE DIFFÉRENCIÉ SOUS LA} GRILLE. - Des tentatives sont faites pour augmenter la tension de claquage dans les MESFETs et les TEGFETs en utilisant un profil de dopage différencié sous la grille, très faible à son contact et élevé à distance. La couche de faible dopage au contact de la grille présentant une ionisation moindre devrait, en quelque sorte, jouer un rôle un peu analogue à celui de la couche d'isolant de la structure MISFET. Pour notre part, nous en avons fait l'expérience dans le cas du TEGFET avec la structure schématisée sur la figure 11, utilisant une épaisseur variable entre 0 et $400 \AA$ de $\mathrm{AlGaAs}$ non intentionnellement dopée (NID). La modélisation bidimensionnelle sans courant a donné des tensions de claquage de 14,2, 14,6 et $12,5 \mathrm{~V}$ pour, respectivement, des épaisseurs de 0 , 200 et $400 \AA$ ce qui fait que l'intérêt de cette technique n'est pas toujours démontré. Ces résultats théoriques ont été corroborés par les mesures effec- 

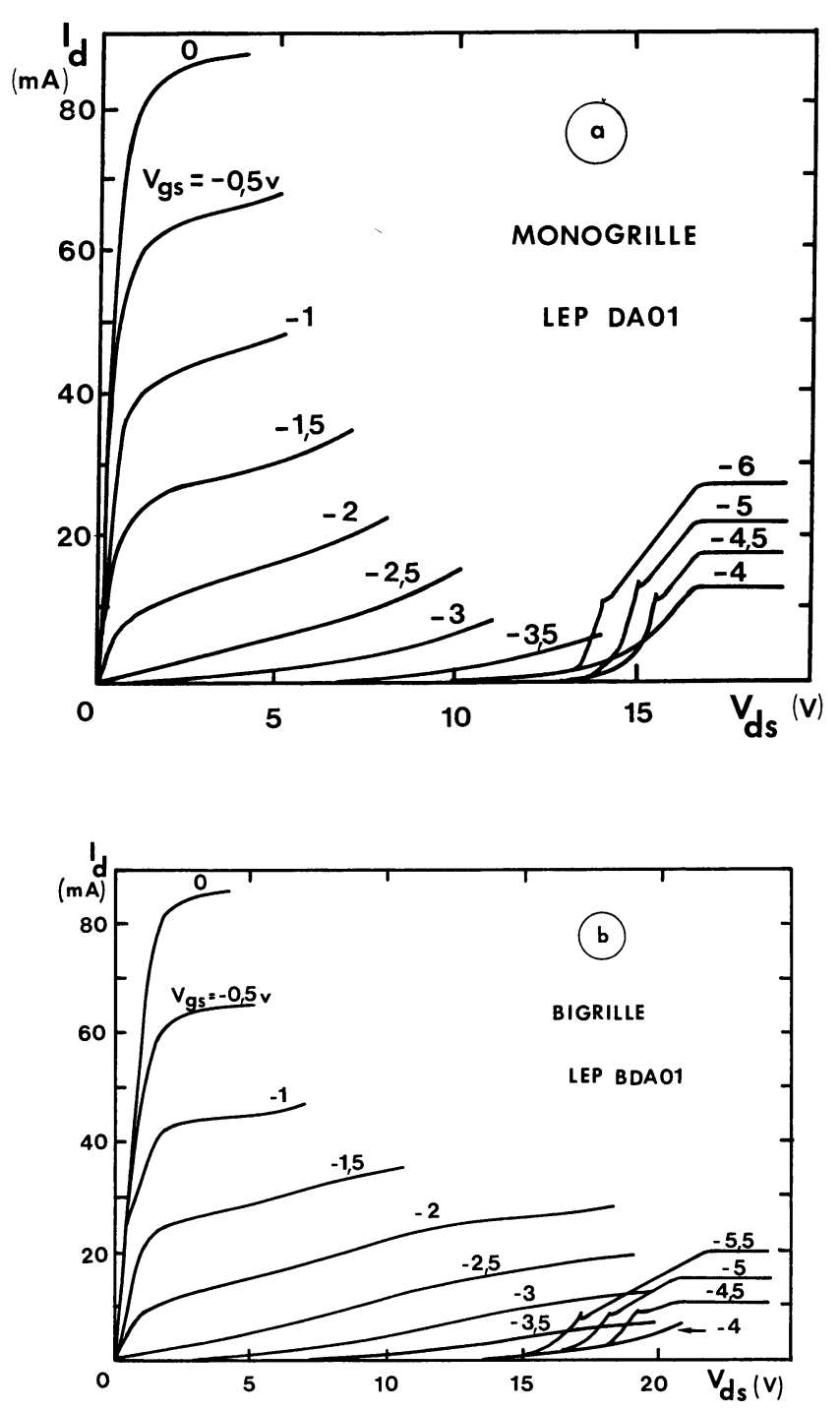

Fig. 9. - Evolution expérimentale du courant drain $I_{\mathrm{D}}$ en fonction de la tension $V_{\mathrm{DS}}$ pour différentes tensions $V_{\text {GS }}$ pour un MESFET monogrille (a) et un MESFET bigrille (b) ayant des topologies et structures de couches similaires.

[Experimental dependence of the drain current $I_{\mathrm{D}}$ versus the $V_{\mathrm{DS}}$ voltage for different $V_{\mathrm{GS}}$ for a single-gate (a) and a dual-gate (b) MESFET's exhibiting similar topologies and layer structures.]

tuées sur 4 échantillons dont l'épaisseur d'AlGaAs NID a été modulée par la profondeur du recess de grille : ces mesures montrées sur la figure 11, donnent, en effet, des résultats très voisins.

L'explication de ces résultats apparaît immédiatement à l'analyse des configurations des lignes de champ électrique dans la structure. C'est ce que montre la figure 12 correspondant à une structure TEGFET dont la couche de AlGaAs comporte une couche NID de $200 \AA$ sur une couche dopée à $2 \times 18^{18} \mathrm{~cm}^{-3}$ de $400 \AA$. L'évolution de l'amplitude

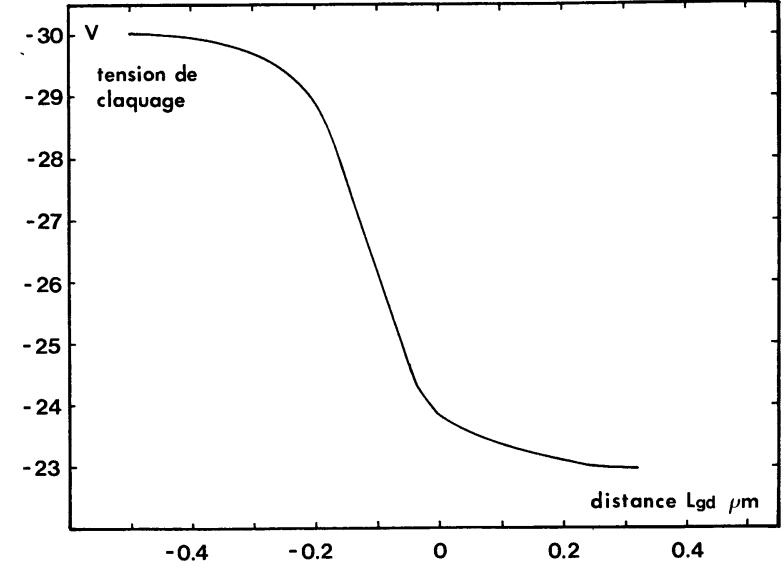

Fig. 10. - Evolution théorique de la tension de claquage d'un MISFET InP en fonction de la distance grille-drain.

[Theoretical dependence of the breakdown voltage on the gate-drain length for an InP MISFET.]

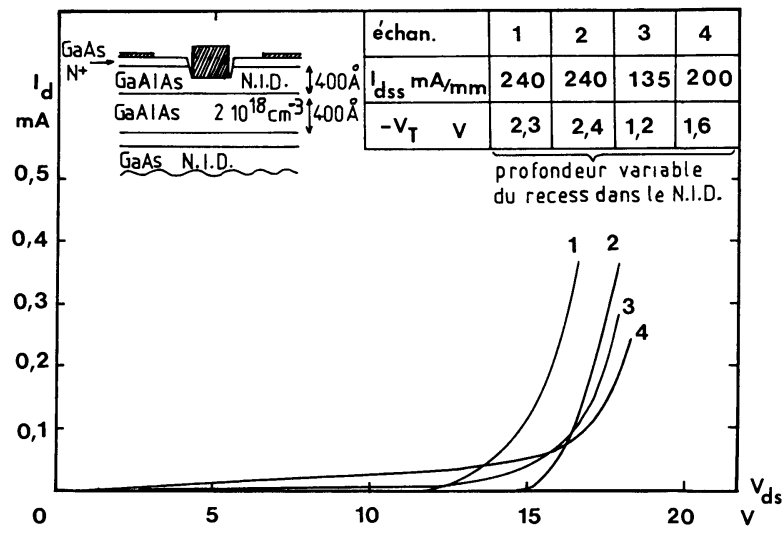

Fig. 11. - Exemples de claquages pour quatre structures TEGFET à profil de dopage différencié pour la couche de AlGaAs, la profondeur de recess étant variable.

[Examples of breakdown voltages for four TEGFET structures using a AlGaAs differentiated doping layer and different recess depths.]

du champ électrique, en fonction de l'abscisse curviligne, est donnée pour l'axe de symétrie de la structure et trois lignes de champ proches du bord de grille, la ligne (2) étant celle satisfaisant à la condition d'avalanche. On remarque que si la couche NID joue pleinement son rôle de tampon sur l'axe de symétrie, en contenant le champ électrique à des valeurs raisonnables, il n'en est pas du tout de même pour les lignes de champ proches du bord de grille car le champ électrique y atteint des valeurs très élevées uniquement à cause des effets fortement 

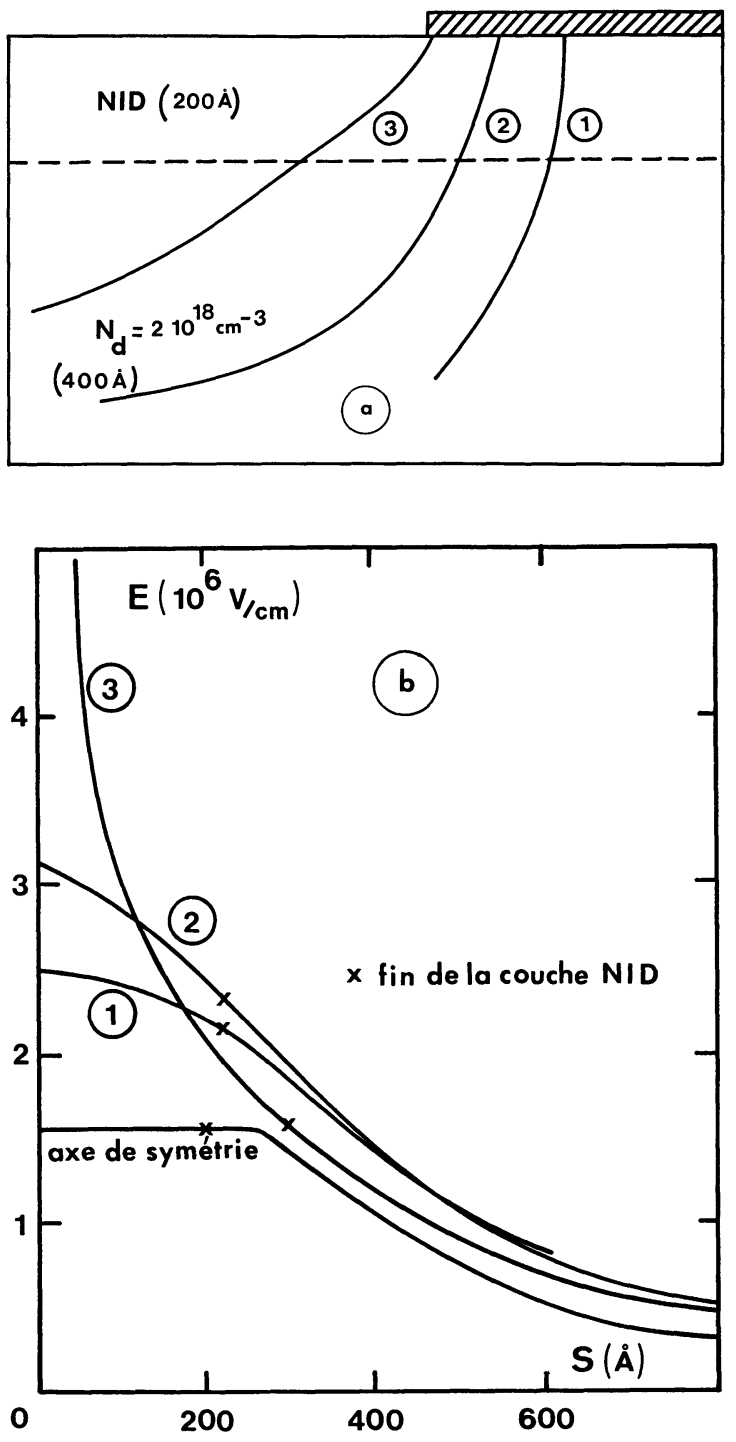

Fig. 12. - Evolution du champ électrique pour différentes lignes dans le cas du TEGFET utilisant une couche NID sous la grille.

[Electric field dependence for different lines for the TEGFET using an undoped layer under the gate.] bidimensionnels qui se produisent dans cette zone. Ces derniers étant étroitement liés à la singularité géométrique du bord de grille, il est évident que l'on doit s'attendre à ce que le claquage puisse être fortement influencé par toute modification de l'aspect de cette singularité géométrique et, par conséquent, que les structures à profil différencié puissent présenter des résultats en claquage très variables, tantôt moyens, tantôt excellents.

\section{Conclusion.}

Les modélisations décrites dans ce travail s'avèrent des outils indispensables pour la conception des transistors à effet de champ. Elles nous ont permis de mettre en évidence l'extrême importance des effets bidimensionnels, la tension de claquage d'un transistor à effet de champ étant essentiellement dépendante de la configuration du champ électrique dans l'espace grille-drain. Les différentes études théoriques ont permis de définir la structure optimale de différents types de transistor permettant d'obtenir des puissances élevées grâce à l'analyse de l'influence des principaux paramètres technologiques dans le but d'obtenir la tension de claquage la plus élevée possible en tenant compte de l'influence d'autres facteurs tels que le courant drain et la transconductance. Elles ont déjà permis soit de corroborer, soit d'interpréter un grand nombre d'observations expérimentales. La comparaison des différentes structures montre que potentiellement la tension de claquage d'un MESFET GaAs est inférieure à celle du TEGET AlGaAs/GaAs, elle-même inférieure à celle du MISFET InP. Nous envisageons, par la suite, de développer les deux types de modélisation proposés afin de pouvoir traiter les technologies les plus difficiles, pour les différentes structures en particulier l'influence d'un recess de grille qui apparaît très souvent comme l'élément déterminant de la tenue au claquage.

\section{Bibliographie}

[1] Wroblewski, R., Salmer, G. and Crosnier, Y., IEEE Trans. Elec. Dev. 30 (1983) 154.

[2] Frensley, W. R., IEEE Trans. Elec. Dev. 28 (1981) 962.

[3] Wemple, S. H., Niehaus, W. C., Cox, H. M., Dilorenzo, J. V. and SCHLOSSER, W. O., IEEE Trans. Elec. Dev. 27 (1980) 1013.

[4] Zaitlin, M. P., IEEE Trans. Elec. Dev. 33 (1986) 1635.

[5] Bulman, G. E., Robbins, V. M. and Stillman, G. E., IEEE Trans. Elec. Dev. 32 (1985) 2454.
[6] Shabde, S. N. and YeH, J. Appl. Phys. 41 (1970) 4743.

[7] Chung-Whei Kao and Crowell, C. R., Solid-State Elect. 23 (1980) 881.

[8] Chen, I. C., Holland, S. and Hu, C., IEEE Elec. Dev. Lett. 7 (1986) 164

[9] El-SAyed, O. L., El-GhazAly, S., SAlmer, G. and LEFEBVRE, M., Solid-State Elect. 30 (1987) 643.

[10] Mizuta, H., Yamaguchi, K. and Takahashi, S. IEEE Trans. Elec. Dev. 34 (1987) 2027. 